\title{
Más Allá del Paradigma de la Alfabetización. La Adquisición de Cultura Científica como Reto Educativo
}

\author{
Irene Díaz ${ }^{(1)}$ y Myriam García ${ }^{(2)}$ \\ (1) Unidad de Investigación en Cultura Científica, Centro de Investigaciones Energéticas, \\ Medioambientales y Tecnológicas (CIEMAT), Av. Complutense, 22, Madrid-España \\ (e-mail: irene.diaz@ciemat.es) \\ (2) Departamento de Filosofía, Universidad de Oviedo, c/ Teniente Alfonso Martínez s/n \\ Oviedo-España (e-mail: garciamyriam.uo@uniovi.es)
}

Recibido Dic. 30, 2010; Aceptado Feb. 07, 2011; Versión final recibida Feb. 24, 2011

\begin{abstract}
Resumen
Este trabajo analiza algunos de los aspectos más significativos que, para el contexto educativo, ha puesto de manifiesto la tradición de los estudios sobre Ciencia, Tecnología y Sociedad (CTS). Dicha aproximación ha modificado significativamente la concepción de la ciencia y la tecnología, aunque el paradigma educativo en la enseñanza científico-tecnológica continúa manejando el modelo positivista anterior. Esto ha producido un desajuste significativo entre la ciencia y la tecnología que se presenta en las aulas, y las que el alumnado necesita. El trabajo concluye que la propuesta permitirá proporcionar de manera más adecuada las competencias necesarias para que el alumnado se desenvuelva en el actual contexto tecnocientífico.
\end{abstract}

Palabras clave: estudios CTS, cultura científica, alfabetización, educación científico-tecnológica,

\section{Beyond the Paradigm of Literacy. Acquisition of Scientific Culture as an Educative Goal}

\begin{abstract}
This paper discusses some of the most significant aspects that in the educational context have emphasized the tradition of the so-called Science and Technology Studies (STS). The STS have significantly changed the conception of science and technology, but the educational paradigm in science and technology teaching continues to manage the old positivist model. This has produced a significant gap between science and technology presented in the classroom and those that students actually need. It is concluded that the notion of scientific culture proposed will better provide the skills that students need to live in the present techno-scientific world.
\end{abstract}

Keywords: STS studies, scientific culture, literacy, scientific and technologic education 


\section{INTRODUCCIÓN}

En las últimas décadas del siglo $\mathrm{XX}$, han sido muchos los intentos por delimitar las características definitorias de nuestras sociedades contemporáneas. Entre ellas, sin duda, destaca la enorme repercusión que tienen la ciencia y la tecnología, sus desarrollos y potenciales riesgos (Beck, 1986). Una de las consecuencias de esta creciente importancia es la preocupación institucional por la alfabetización y la capacitación científico-tecnológica de la ciudadanía, con el objetivo de responder a las expectativas y necesidades de una sociedad cada vez más compleja. En este sentido, un testimonio privilegiado puede encontrarse en la Declaración final del Congreso Mundial sobre Ciencia celebrado en Budapest (Hungría) en 1999 bajo el lema "Ciencia para el siglo XXI, un nuevo compromiso". En ella, se recoge la necesidad de localizar nuevas vías para que la ciudadanía tenga acceso adecuado a los conocimientos científicos (UNESCO, 1999). Desde entonces, las políticas públicas han prestado una atención prioritaria a mejorar la capacidad de los sistemas de educación para garantizar la calidad de las enseñanzas y satisfacer la demanda de profesionales cualificados.

Más recientemente, y en el contexto concreto de la región iberoamericana, el documento Metas 2021 (OEl, 2010) reitera el compromiso con el fomento de una adecuada educación científicotecnológica (Meta específica 12) y el refuerzo de la cooperación científica, tecnológica y de la innovación (Meta específica 23). Queda patente, por tanto, que la búsqueda de una mejor orientación educativa para la enseñanza de estas materias continúa siendo una necesidad de primer orden, cuyo ambicioso objetivo se sitúa ante la exigencia de dar cuenta no solo de una serie de contenidos e informaciones originados en las disciplinas científicas, sino de proporcionar además a la ciudadanía del futuro las herramientas precisas para desenvolverse en un contexto de cambios rápidos y globales, mediados por los desarrollos científico-tecnológicos -otras investigaciones al respecto que cabe mencionar son las recogidas en Abell y Lederman (2007), la desarrollada por Holbrook y Rannikmae (2009) o, más específicamente centrada en la evolución de los modelos educativos en la enseñanza de las ciencias, Duschi (2008).

Por nuestra parte, y tomando como marco de análisis los estudios sociales de la ciencia y la tecnología (o estudios CTS), examinaremos cómo estas necesidades no podrán ser satisfechas desde la esfera educativa mientras sigan dominando los esquemas de enseñanza tradicionales y sus rígidas divisiones entre el ámbito de los hechos y de los valores, entre la clase de ciencias y la clase de humanidades y, en definitiva, la división del conocimiento en dos esferas o culturas separadas entre sí (López, 2005). Este trabajo, por tanto, aportará una perspectiva complementaria, centrada en la cultura científica, a la línea más general de reflexión sobre la educación desarrollada desde los estudios sociales de la ciencia por autores como De Arriba (2009) -centrado en la educación para adultos-, Howe (2009) -quien focaliza su atención en torno al modelo de ciencia subyacente- o Mansour (2009), que se orienta hacia el papel docente-.

\section{LA CIENCIA Y LA TECNOLOGÍA EN EL MARCO EDUCATIVO IBEROAMERICANO}

Más de cincuenta años después de que Snow (1959) pronunciase su famosa conferencia sobre la existencia de dos culturas aparentemente confrontadas (la científica, objetiva, y la humanística, subjetiva), la brecha parece permanecer abierta. Más aún, en cierto sentido, se ha incrementado. En efecto, no solo podemos hablar ya de la incomunicación entre una y otra culturas, hecho al que Snow hacía referencia-, sino también de una muy diferente concepción con respecto a la dinámica y apropiación de una y otra, que se traslada a los contextos educativos.

Cuando en torno al siglo XVII se produjo la primera Revolución científica (siguiendo a Echeverría, 2003), la ciencia comenzó a cobrar una importancia creciente en las sociedades, hasta alcanzar el papel central que ocupa en nuestros días. A partir de la Revolución industrial del siglo XIX, la tecnología sigue un proceso similar que le ha otorgado actualmente un papel hegemónico en nuestras vidas. De este modo, ciencia y tecnología constituyen actualmente dos instituciones altamente vinculadas con el desarrollo económico y social de los países. Por ello, una de las preocupaciones significativas, dentro del panorama político actual de toda la región, es atender no 
solo al fomento de la I+D+i y al número de recursos materiales y humanos relacionados con ella, sino también a la formación de los futuros profesionales (OEI, 2010; UE, 2010).

Además, en sociedades cada vez más permeadas por la ciencia y la tecnología, el conocimiento científico-tecnológico se torna en herramienta imprescindible para el adecuado desempeño de la ciudadanía. En este contexto un conocimiento memorístico, tal y como ha venido siendo concebido tradicionalmente, se muestra insuficiente; es necesaria una apropiación significativa del mismo que permita valorar el desarrollo, no solamente en términos de mejoras y crecimiento, sino también de sus potenciales riesgos para el entorno, la posibilidad de que acreciente la brecha económica entre países, la existencia de dilemas éticos o valorativos, etc. De este modo, la formación adecuada de una ciudadanía capaz, responsable y autónoma, que pueda tomar decisiones conscientes e informadas, se constituye como un verdadero reto social.

Por supuesto, y en ambos casos, la formación requerida -tanto para los futuros científicos como para una ciudadanía capaz de desenvolverse en las sociedades contemporáneas- no puede obtenerse únicamente en el contexto del sistema educativo, si bien es precisamente en él donde comienza y donde se hace necesario además sentar las bases que posibiliten posteriormente el autoaprendizaje y la actualización de esos conocimientos mediante informaciones que pueden proceder de fuentes diversas (medios de comunicación, museos, campañas institucionales, y ONGs, entre otras). De ahí la importancia que los gobiernos conceden a la formación científica y tecnológica dentro de las aulas en todos los niveles.

\section{Retos educativos sobre ciencia y tecnología}

La formación que, de manera genérica, se imparte en las aulas suele tener varios objetivos fundamentales que son compartidos a nivel tanto regional (entendiendo en este sentido la región iberoamericana) como en un contexto internacional más amplio. Nos referimos, por ejemplo, a la necesidad de una adquisición comprensiva de conocimientos, a la capacitación del alumnado para la utilización de nuevas herramientas y recursos -especialmente digitales-, al fomento de las vocaciones científicas, a la comprensión de la necesidad de mantener una relación más armónica con el entorno o a la formación de una ciudadanía que valore, de manera crítica y responsable, el desarrollo científico y tecnológico. Por tanto, es posible afirmar que, del mismo modo que la lucha contra el analfabetismo fue sin duda el objetivo educativo de la llustración, para las sociedades contemporáneas la generación de cultura científica se ha convertido en un reto fundamental.

Han sido variadas las iniciativas que, en el marco educativo, se han emprendido durante los últimos años para tratar de satisfacer esta necesidad. Por ejemplo, en el caso de España, durante las últimas dos décadas se han desarrollado e implementado dos reformas educativas para las etapas previas a la formación universitaria -la Ley Orgánica de Ordenación General del Sistema Educativo (LOGSE) en 1990 y la Ley Orgánica de Educación (LOE) en 2006- que han tratado de satisfacer los objetivos mencionados mediante la inclusión de asignaturas concretas en el currículo (por ejemplo, la asignatura Ciencia, tecnología y sociedad en la LOGSE o Ciencias para el mundo contemporáneo en el caso de la LOE). Además, se han destinado partidas económicas específicas dirigidas a la adquisición de recursos informáticos o a la mejora de los ya disponibles en los centros, al desarrollo de materiales, plataformas digitales y contenidos didácticos que apoyen el trabajo en el aula, o a la financiación de proyectos de investigación e innovación educativa. Por su parte, los diferentes Organismos Nacionales de Ciencia y Tecnología (ONCYTs) de la región iberoamericana e instituciones con actividad transnacional como la OEI han venido impulsando también estudios y análisis acerca de las peculiaridades educativas de cada país, programas de formación docente, desarrollo de materiales específicos, etc. Todo ello, con el objetivo final de tratar de capacitar más adecuadamente al profesorado y mejorar los resultados de aprendizaje del alumnado iberoamericano en el campo concreto de la ciencia y la tecnología.

Sin embargo, el rendimiento de las medidas mencionadas, pese a haber dado como resultado algunas mejoras notables, parece haber tenido un éxito limitado. Así queda recogido, por ejemplo, en el reciente Informe PISA de 2006 (OCDE, 2008), donde los resultados obtenidos por el alumnado iberoamericano no responden, en términos generales, a las expectativas de las 
iniciativas mencionadas. Por supuesto, no se pueden generalizar una serie de aspectos concretos que permitan abarcar la enorme variabilidad de problemáticas presentes en la educación científica y tecnológica de Iberoamérica, dada la gran diversidad de países y regiones que la constituyen. Con todo, cabe destacar la existencia de una concepción positivista de la ciencia, la tecnología y la cultura científica que subyace a la enseñanza de las materias correspondientes y que, en nuestra opinión, condiciona los resultados de enseñanza-aprendizaje y la efectividad de las políticas educativas adoptadas.

\section{CONCEPCIÓN DE LA CIENCIA Y LA TECNOLOGÍA}

Afirman Funtowicz y Ravetz (1993) que "la ciencia evoluciona en la medida en que es capaz de responder a los principales desafíos de cada época, cambiantes a través de la historia". En efecto, desde los estudios CTS ha sido posible identificar una serie de revoluciones, la última de las cuales habría tenido lugar en torno a los años 80 del siglo XX (Echeverría, 2003), consolidando lo que ha venido a denominarse un nuevo modo de producción de conocimiento. La denominación y caracterización precisas para ese nuevo modo de producción no ha alcanzado un consenso específico, pudiendo encontrarse un amplio mosaico de aproximaciones (especialmente, Funtowicz y Ravetz, 1993; Gibbons et al., 1994; Jasanoff, 1995; Ziman, 2003; Echeverría, 2003; precedidos todos ellos por los trabajos de Weinberg, 1972) y denominaciones, tecnociencia, ciencia posnormal, ciencia reguladora. Con todo, existe un acuerdo sobre la existencia de una nueva producción de la ciencia y la tecnología que presenta diferencias significativas en relación a sus predecesoras surgidas en la Modernidad y que reclama, en consecuencia, una nueva manera de evaluarlas, gestionarlas, relacionarlas con la sociedad y, desde luego, aprehenderlas y trasladarlas a las aulas.

De manera resumida, de acuerdo con la imagen de la ciencia procedente del empirismo lógico de principios del siglo XX -o concepción heredada, siguiendo a Putnam (1962), esta es el resultado de la aplicación de un método racional (el denominado método científico) a partir de una observación directa de la naturaleza y con el complemento de la experimentación. Según este modelo, la ciencia ha sido capaz de elaborar todo un edificio de conocimiento racional, objetivo, fiable, autónomo y libre de valores no epistémicos que, además, revierte en beneficios sociales y permite contar con el asesoramiento experto de los científicos y tecnólogos para la toma de decisiones políticas. Bajo esta concepción, el paradigma de la "buena ciencia" vendría ejemplificado por la física newtoniana, que toma la matemática como herramienta operatoria, la lógica como estructura de base y el empirismo clásico (heredado de las aportaciones de autores como Descartes, Locke o Hume) como marco filosófico de referencia. Asociada a esta visión tradicional, la tecnología fue objeto de una concepción estrecha que la describió como mera ciencia aplicada donde el valor canónico se corresponde con la eficiencia. Por otro lado, y en tanto que aplicación de una ciencia considerada valorativamente neutral, la tecnología misma no se considera tampoco influida por valores externos, lo que impide que sea juzgada éticamente más allá de la evaluación de lo que con sus desarrollos hagan los seres humanos.

Ambas imágenes idealizadas tienen un punto de inflexión en el entorno académico con la publicación de La estructura de las revoluciones científicas de Thomas Kuhn (1962). En esta obra, Kuhn presenta una dinámica científica alejada del modelo tradicional y que, sumada a otros problemas -internos, como los relativos a los términos disposicionales; o externos, como la carga teórica de la observación o la infradeterminación-, provoca una reorientación en la concepción académica de la ciencia y la tecnología. Será en este contexto donde aparezcan los estudios CTS, que "han construido una nueva imagen de la ciencia y la tecnología, con bastante coherencia y un gran poder imaginativo y práctico" (Edge, 1995). Según este mismo autor, lo más relevante que ha aportado esta nueva perspectiva ha sido una humanización de la ciencia, entendida no como un producto de los seres humanos (los científicos, que serían, por tanto, falibles, interesados, etc.), sino como un logro del ingenio humano. La ciencia y la tecnología comienzan entonces a ser descritas como procesos sociales caracterizados por la presencia de valores de todo tipo (Echeverría, 2003; Jasanoff, 1995; Ziman, 2003), vinculados a la incertidumbre y el riesgo (Winner, 1986) y donde existen lagunas epistémicas y controversias entre expertos (Funtowicz y Ravetz, 1993). 
A grandes rasgos, pueden mecionarse tres grandes cambios en la aproximación metacientífica a raíz de esta nueva perspectiva. En primer lugar, el reconocimiento de la necesidad de realizar un estudio interdisciplinar y crítico acerca de la ciencia y la tecnología, que incluya aspectos que han venido quedando relegados por considerarse externos a este ámbito: los condicionantes sociales. En segundo lugar, el acento puesto en la consideración de la ciencia como una dinámica -y no un mero producto- que, por tanto, se determina mediante un proceso evolutivo. Finalmente, la cada vez mayor vinculación existente entre la ciencia y la tecnología, así como la constatación de que se difumina de manera progresiva la frontera tradicionalmente bien consolidada entre hechos y valores, que permitía establecer un lugar seguro para el conocimiento científico dentro del esquema positivista, más allá de consideraciones valorativas, intereses políticos, sociales. De este modo, los estudios CTS han tratado en las últimas décadas de desmitificar la tradicional concepción de la ciencia y la tecnología y de llevar estas ideas al ámbito educativo, siendo una de sus preocupaciones destacadas ya desde sus inicios la atención a la formación de los científicos e ingenieros (Edge, 1995).

\section{LAS CONCEPCIONES DE LA CULTURA CIENTÍFICA}

Los cambios operados en la conceptualización académica de la ciencia y la tecnología desde el campo de los estudios sociales de la ciencia, han tenido además una repercusión significativa en relación a la comprensión de la cultura científica y sus dimensiones. Así, partiendo de la imagen tradicional asociada a la concepción heredada, cultura científica y alfabetización son prácticamente sinónimas (ya que la ciencia es un producto acabado, fiable, estable, etc. cuyo aprendizaje equivale a una especie de adiestramiento conceptual y teórico de sus logros más relevantes). Ahora bien, al modificarse la imagen de la ciencia y la tecnología, e introducirse en ella las dimensiones sociales, es necesario replantear también el paradigma de la cultura científica. Una revisión de las principales propuestas conceptuales y metodológicas permitirá comprender las cuestiones clave de la investigación en este campo durante los últimos treinta años. En esta línea, Bauer et. al. (2007) realizan una síntesis de los distintos tres paradigmas que han guiado la investigación al respecto.

\section{La alfabetización científica}

La idea de alfabetización científica, que toma su definición de la noción genérica de alfabetización, tiene su origen en el siglo XIX. Históricamente, un individuo se consideraba letrado si podía leer y escribir su propio nombre; a partir del siglo XIX, ser alfabeto significa ser capaz de leer y escribir. Basándose en este concepto, la alfabetización científica podría definirse como la capacidad de leer y escribir sobre ciencia y tecnología (Miller, 2000). Ahora bien, dada la amplia gama de aplicaciones científicas y técnicas en la vida cotidiana, Shen (1975) sugiere que la comprensión pública de la ciencia y la tecnología puede ser útilmente dividida en alfabetización científica práctica, alfabetización científica cultural y alfabetización científica cívica.

La alfabetización científica práctica permite a un individuo hacer frente a los problemas básicos de supervivencia. Tiene que ver, por tanto, con cuestiones tales como la vivienda, el agua y los alimentos, la dieta, la salud y la crianza de los hijos. La alfabetización científica cultural tiene que ver con el reconocimiento y apreciación de la ciencia como un logro majestuoso de la inteligencia y el espíritu humano. La alfabetización científica cívica permite a un ciudadano contribuir en los debates sobre cuestiones relacionadas con la ciencia que afectan a una sociedad.

En este contexto, la alfabetización científica cívica se define como el nivel de comprensión de la ciencia y la tecnología necesario para funcionar como ciudadanos en una sociedad industrial (Miller, 2000). La propuesta de Miller distingue, a su vez, cuatro elementos: el conocimiento de los hechos básicos de la ciencia, la comprensión de los métodos científicos (como el razonamiento de probabilidad y el diseño experimental), una estimación positiva de los resultados de la ciencia y la tecnología para la sociedad, y el rechazo de las creencias supersticiosas como la astrología o la numerología (Bauer et al., 2007). 
La referencia clásica y principal sobre comprensión y actitudes públicas hacia la ciencia y la tecnología la representa la National Science Foundation (NSF) que incluye desde hace más de treinta años la propuesta inicial de Miller. En general, se argumenta que la combinación de un nivel razonable de logros en cada una de estas dimensiones reflejará un nivel de competencia suficiente para comprender y seguir los argumentos sobre los asuntos políticos de ciencia y tecnología en los medios de comunicación. De este modo, la definición de alfabetización científica propuesta por Miller se constituye como una medida de umbral: para calificar a un miembro del público como "atento a la ciencia" se requiere que maneje un nivel mínimo de alfabetización, que se interese y se sienta informado sobre ciencia y tecnología, que aprecie sus resultados positivos, y renuncie a las supersticiones. Sobre la base de esta medida, no más de una cuarta parte de encuestados son calificados como científicamente alfabetizados (Sturgis y Allum, 2004). Ante tales evidencias empíricas, el público se caracteriza por un déficit cognitivo. Además, la interpretación de los datos llevada a cabo por Miller apunta a una fuerte asociación entre la educación formal y la alfabetización científica.

Asumido el modelo de déficit cognitivo, y como consecuencia de este tipo de interpretaciones, las intervenciones propuestas en el marco de este paradigma pertenecen principalmente al campo de la educación, con vistas a aumentar el nivel básico de alfabetización. En el terreno político, se asume una actitud tecnocrática: si la inmensa mayoría de la población adulta estadounidense es científicamente analfabeta, la conclusión que se deriva es que será casi imposible que sean capaces de seguir el debate público sobre los resultados científicos o las cuestiones de política pública relacionadas con la ciencia y la tecnología.

\section{La comprensión pública de la ciencia}

En la segunda mitad de la década de 1980, las nuevas preocupaciones surgen bajo el título de Public Understanding of Science (PUS). En el Reino Unido esta transición está marcada por la publicación del informe de la Royal Society (1985), encargado en la creencia de que el interés del público por la ciencia y el apoyo a los científicos se esfumaba. El informe sugiere no sólo que los científicos tienen la obligación de salir y comunicar los beneficios de la ciencia, sino también que una ciudadanía científicamente alfabetizada será más favorable a los programas de investigación científica y más entusiasta con las innovaciones tecnológicas. De este modo, la preocupación por la alfabetización científica es trasladada dentro del paradigma PUS. Ahora, la diferencia estriba en la atención central que se prestará a las actitudes, bajo el axioma "cuanto más lo conoces, más lo quieres", construido alrededor de la convicción de que la ciudadanía sabe poca ciencia (Bauer et al., 2007). De acuerdo con el informe, el resultado esperable de las nuevas iniciativas de divulgación científica será un mayor apoyo social a los programas de investigación por parte de un público que ahora ya contará con un nivel suficiente de información (Sturgis y Allum, 2004).

En el plano de la investigación, la correlación entre conocimiento y actitud se convierte en el foco de interés. En general, se supone que el interés representa una base fiable para medir la comprensión y la formación de actitudes de un individuo: las personas que están interesadas en un tema en particular tienden a estar relativamente bien informadas al respecto. Sobre la base de esta hipótesis, la investigación llevada a cabo en los años 80 y 90 muestra que los encuestados que declaran estar muy interesados y muy bien informados obtienen mejores resultados en evaluaciones objetivas de los conocimientos científicos. Aceptada esta hipótesis, la consecuencia evidente para la política científica será la movilización de campañas de información pública orientadas a remediar el desinterés del público por la ciencia. Así, las intervenciones propuestas en el marco de este paradigma pertenecen principalmente al área de la comunicación social. En la actualidad, numerosos trabajos siguen realizándose sobre la base de que el conocimiento es un determinante fundamental de las actitudes de los ciudadanos, y esta es también en buena medida la concepción subyacente en la enseñanza de las disciplinas científico-tecnológicas.

\section{Ciencia y Sociedad}

A partir de la década de 1990 el foco de atención pasará a centrarse en el déficit de las instituciones científicas y de los expertos, que adoptan prejuicios sobre un público al que consideran ignorante (Bauer et al., 2007). Como consecuencia de este nuevo déficit, la opinión 
pública de los científicos se convierte en objeto de escrutinio y se atiende a nuevas dimensiones y factores, como la confianza, en la relación ciencia-sociedad.

La correlación entre la confianza y el apoyo a la investigación se evidencia en el Eurobarómetro 39.1 (UE, 1993) sobre percepción pública de la biotecnología en la Unión Europea. Mientras que en Dinamarca y Alemania (dos de los estados europeos más industrializados), el público tiende a juzgar como bastante alto el riesgo asociado al uso de la tecnología del ADN recombinante, el público danés parece bastante más dispuesto que el alemán a apoyar la continuación del uso de dicha tecnología. La pregunta aquí es ¿por qué las personas que comparten una visión común de una tecnología como algo arriesgado, difieren en su voluntad de desarrollarla? Una posible respuesta puede deducirse de los resultados de otra pregunta presente en el Eurobarómetro, que pidió a los entrevistados que clasificasen el grado de confianza que sentían hacia diferentes instituciones que podían proporcionar una información fiable sobre ciencia y tecnología. Los resultados a esta pregunta revelan que son los grupos ecologistas y las organizaciones de consumidores las instituciones más confiables, mientras la industria y el gobierno se sitúan en los últimos lugares. Atendiendo específicamente a los datos de Alemania y Dinamarca, el público danés muestra una mayor confianza que el público alemán en la fiabilidad de sus autoridades públicas como fuente de información sobre la biotecnología; es decir, las personas que tienen una mayor confianza en las instituciones responsables de la gestión de una tecnología de riesgo están más dispuestas a desarrollar dicha tecnología.

Este tipo de datos refleja la cambiante relación entre la ciencia y el público. Ya no se trata simplemente de tratar con el conocimiento y la ignorancia, sino también con la confianza y la desconfianza, y, sobre todo, con su ambivalencia (Durant, 1995). Esta tendencia se puede identificar igualmente en otros estudios sobre percepción social de la ciencia y la tecnología a nivel iberoamericano, el más reciente de todos ellos desarrollado en el año 2007 en siete grandes ciudades de Iberoamérica (FECYT, OEI, RICYT, 2009)-.

Las acciones propuestas en el marco de este paradigma tendrán como objetivo reorientar las instituciones y la política a fin de reconstruir la confianza pública y atender a las actitudes negativas que evidencian las encuestas a gran escala, la investigación de grupos focales y las observaciones cuasi-etnográficas, y que han llevado a la declaración de una "crisis de confianza en los ciudadanos". En este contexto, la deliberación pública y la participación serán el nuevo camino a tomar para reconstruir la confianza (Bauer et al., 2007). Ahora bien, si aceptamos la descripción realizada en el apartado anterior sobre la ciencia y la tecnología contemporáneas, la mera alfabetización no proporciona los recursos necesarios para la toma de decisiones y el correcto manejo de la propia vida en entornos tecnocientíficos. Participar en el desarrollo o la gestión de la ciencia y la tecnología no es únicamente tener en cuenta el conocimiento de los aspectos técnicos, sino también los aspectos sociales, políticos, económicos y éticos relacionados.

\section{MÁS ALLÁ DEL PARADIGMA DE LA ALFABETIZACIÓN}

Tanto el paradigma de la alfabetización científica como el de la comprensión pública de la ciencia asumen un estado público de deficiencia. El modelo de déficit parte de la asunción de que el conocimiento científico constituye un cuerpo reconocible de información codificada, de modo que es posible medir la cantidad de información incorporada por un individuo y establecer su grado de déficit en la comprensión de la misma. En primer lugar, es posible afirmar que, probablemente, los especialistas no se pondrían fácilmente de acuerdo acerca de cuáles deberían ser los contenidos cognitivos que habitualmente se incluyen en el objeto transferido; esto es, los contenidos específicos indispensables que el público no puede ignorar. En segundo lugar, la definición propuesta por Miller establece una asociación directa entre alfabetización científica y comprensión de los métodos de la ciencia y algunos contenidos específicos de conocimiento general (así, por ejemplo, la creencia en la astrología descalifica a cualquiera de ser científicamente alfabetizado). Lo que cuenta como conocimiento científico es, en última instancia, lo que Miller sugirió en sus dimensiones: los hechos y los métodos. Las diferencias entre el conocimiento científico y el conocimiento del público serán interpretadas en términos de ignorancia y, bajo estos cánones, no 
es extraño que los indicadores arrojen resultados previsibles de baja comprensión (Bauer et al., 2007). A partir de este tipo de consideraciones, una creciente literatura crítica inspirada en los estudios sociales de la ciencia ha puesto de manifiesto que la conceptualización tradicional y los instrumentos de medida habitualmente utilizados en estos documentos tienen un valor limitado, siendo ampliamente criticados (Godin y Gingras, 2000).

El primer gran problema que se identifica es que definen y miden la cultura científica principalmente a través del conocimiento de los hechos. Wynne (1995) observa que la cultura científica es entendida como forma de instrucción, de acumulación del saber, sea éste socialmente válido o no. A esta concepción subyace la idea de ciencia de la concepción heredada, expuesta en los apartados anteriores. De acuerdo con esta imagen tradicional, la ciencia es entendida como un cúmulo coherente de conocimientos que describen la naturaleza real de las cosas, y en el que hay poco margen para la duda o el error. En este sentido, cultura científica y alfabetización científica quedan asimiladas. La cultura científica de los individuos se limita a contener los conocimientos básicos de la ciencia cristalizada, es decir, respuestas apropiadas a las preguntas habituales sobre el origen del oxígeno terrestre o la estructura del Sistema Solar.

Esta concepción enciclopédica de cultura científica descuida algunas dimensiones relevantes en el proceso real de transferencia de conocimiento. Aquellas relacionadas, por un lado, con el papel activo del sujeto, que no es un mero receptor pasivo de elementos cognitivos; y, por otro lado, con el componente comportamental del receptor de dichos contenidos (López y Cámara, 2009). Además, no se correspondería ya con la nueva imagen de la ciencia y la tecnología contemporáneas, tal y como han sido definidas por los autores más representativos del campo de los estudios sociales de la ciencia y la tecnología expuestos al principio de este trabajo.

\section{Hacia una concepción amplia de cultura científica}

Lo que los nuevos enfoques críticos señalan es que, en los procesos de transferencia del conocimiento, los receptores no son agentes pasivos sino actitudinalmente activos que utilizan estrategias para filtrar y economizar las informaciones que reciben. Mantienen un sistema propio de creencias y actitudes en el que deben integrar esos elementos cognitivos. Se trata de factores psicológicos relativos a la confianza o a las connotaciones emocionales que pueden acompañar a los elementos informativos relacionados con ciertos temas complejos y conflictivos. Así entendida, la adquisición de cultura científica va mucho más allá de un mero proceso de desarrollo de contendidos, que se demuestra insuficiente y limitado (López y Cámara, 2009).

Sobre la base de la investigación empírica en sucesivas encuestas de percepción social de la ciencia, Cámara y López (2008) defienden que la adquisición de cultura científica consiste no sólo en el "enriquecimiento cognitivo [del individuo] sino también en el reajuste de su sistema de creencias y actitudes, especialmente en la generación de disposiciones al comportamiento basadas en información científica". En este sentido, la disposición al comportamiento o la inclinación a la participación ciudadana, tanto en situaciones ordinarias de la vida como en situaciones extraordinarias, puede considerarse como una dimensión comportamental de la cultura científica. Es decir, atiende a la acción del ciudadano al intervenir en asuntos públicos mediante su opinión, voto o de otros modos, coordinando su acción con las de otros actores a fin de alcanzar cierta meta.

Otra importante crítica que suele hacerse con respecto a los contenidos que se incluyen en el proceso de transferencia es la omisión de contenidos metacientíficos. Cabe esperar que la cultura científica de los individuos no solamente contenga los conocimientos básicos de la "ciencia ortodoxa", sino que también incluya aspectos relativos a los riesgos, efectos adversos, usos políticos, dilemas éticos o influencias económicas de la investigación científica y el desarrollo tecnológico, así como la habilidad o capacidad para hacer frente a los objetos tecnológicos de la vida cotidiana (López y Cámara, 2009). En este sentido, se propone incorporar al repertorio cultural de los individuos los nuevos elementos presentes en la dinámica científico-tecnológica señalados por los estudios sociales de la ciencia. 
Estos nuevos enfoques críticos conducen, en definitiva, a un cambio fundamental en el concepto de cultura científica, que ya no puede considerarse -en una sociedad como la nuestra, cada vez más compleja y tecnificada- de un modo pasivo, atendiendo solamente a la cantidad de información científica asimilada; sino que incluye también la reflexión y saber hacer uso de la información científica para generar opiniones, actitudes críticas y disponer de mejores elementos de juicio en la toma de decisiones. De este modo, implica también una comprensión profunda y significativa de los conocimientos, hasta el punto de convertirlos en herramientas de la propia vida; por ejemplo, a la hora de decidir qué tipo de vehículo comprar o si vacunar a nuestros hijos decisiones que, entre otros criterios como los económicos, estéticos, religiosos, etc. han de tener en cuenta los aspectos científicos y técnicos-. La ciencia y la tecnología dejan entonces de ser meros datos para convertirse en nociones y habilidades apropiadas o aprehendidas.

\section{CONSECUENCIAS PARA EL CONTEXTO EDUCATIVO}

El fenómeno formativo se lleva a cabo a través de dos grandes campos: la educación reglada científico-técnica, y la comunicación social de la ciencia. Ambos campos comparten un mismo objetivo último, la creación de cultura científica, aunque asumiendo distintos roles. Sin embargo, como se acaba de señalar, comunicar con éxito conocimiento científico es un proceso mucho más complejo que una simple cuestión de alcanzar cierto nivel de competencia, registrable mediante preguntas tipo test, y la idea de cultura científica que se maneja corre el peligro de mostrarse limitada y pervierte, desde su origen, el diseño de políticas públicas orientadas a promover la educación científico-tecnológica.

Una concepción de cultura científica limitada y restringida, como la asumida desde el paradigma de la alfabetización, que aún está presente de forma generalizada en las aulas de Iberoamérica, presupone que la ciencia forma parte del acervo cultural de conocimientos con el que todo el mundo debe estar familiarizado. De este modo, la agenda educativa -desde la educación primaria hasta la instrucción de los científicos e ingenieros-, se limita a aumentar sus esfuerzos en relación con una alfabetización básica, entendida en términos de hechos y métodos, que será incapaz de dar cuenta del uso social del conocimiento científico-tecnológico. Una concepción más amplia de la cultura científica hará referencia no solo a ese enriquecimiento cognitivo o alfabetización, sino también al reajuste del sistema de creencias y actitudes, que se reflejan en la generación de disposiciones al comportamiento basadas en información científico-tecnológica (López y Cámara, 2009).

En efecto, partir de una u otra concepción a la hora de abordar la enseñanza implica, no solo diferencias en la vinculación con otras materias educativas y con la sociedad en general, sino también consecuencias relevantes en cuanto a qué enseñar, cómo hacerlo y qué es realmente conocimiento. Es necesario, por tanto, moverse desde una perspectiva enciclopédica cuasi ilustrada, (y ligada a la concepción tradicional de la ciencia y la tecnología), donde el énfasis educativo ha de ser puesto en la alfabetización del alumnado en el sentido más tradicional del término, adquisición de conceptos, memorización de fórmulas, resolución matemática de problemas, hasta una perspectiva que considere que el conocimiento necesario para desarrollar adecuadamente un rol en las sociedades requiere de mucho más que algunos rudimentos conceptuales. El conocimiento, en este contexto, no puede ser medido con un test, ya que la ciencia y tecnología contemporáneas no pueden ser reducidas solo a nociones teóricas, sino que es necesario internalizarlas y ser capaz de emplearlas posteriormente en la vida.

Las razones del escaso éxito de buena parte de las medidas educativas adoptadas en Iberoamérica (inclusión de nuevas materias en el currículo, fomento de programas y ayudas, y introducción de recursos tecnológicos en las aulas, entre otros) se encuentran muy vinculadas al paradigma de alfabetización científica aún dominante. Por ello, consideramos necesario proponer la incorporación en los entornos educativos de una concepción de la cultura científica en la que no se atienda únicamente a su componente alfabetizador, sino también a los aspectos actitudinales y comportamentales. En nuestra opinión, y en consonancia con el panorama descrito, una cultura científica de calidad debe ser crítica y responsable. Ha de incluir conocimiento no solo de las potencialidades de la ciencia sino también de sus incertidumbres, riesgos e interrogantes éticos; 
de su uso político y de la utilidad de este tipo de información para la toma de gran parte de las decisiones cotidianas. Todos estos rasgos han de ser considerados aspectos relevantes a la hora de llevar al aula la enseñanza de las ciencias. Este reto plantea la necesidad de hacer frente a una serie de interrogantes, cuya resolución implicará unas u otras estrategias educativas -desde el planteamiento sobre la utilidad misma de la enseñanza y los aspectos que debe incluir, hasta la reflexión acerca de la inercia acumulada por el sistema educativo y la pertinencia del cambio de modelo (Martín, 2009).

Al respecto, el abandono del paradigma de la alfabetización resulta un reto significativo, ya que para modificarlo es necesario abandonar un lugar bien conocido por el colectivo docente acerca de cómo enseñar. No hemos de olvidar que la mayor parte de la formación de formadores consiste, sobre todo, en la capacitación específica en sus respectivas áreas de conocimiento, que ha sido recibida basándose en los esquemas tradicionales de conceptualización de la ciencia y la tecnología, así como de la didáctica asociada al paradigma de la alfabetización. De este modo, la formación docente se torna un aspecto fundamental para evitar que se llegue a las aulas sin el bagaje teórico adecuado sobre los nuevos marcos de análisis metacientífico. En nuestra opinión, buena parte de las dificultades que los sistemas educativos iberoamericanos están mostrando a la hora de alcanzar los objetivos propuestos para la enseñanza de la ciencia y la tecnología, tienen que ver con esta circunstancia. De este modo, el cambio en el enfoque didáctico y en la conceptualización de base constituyen los pilares fundamentales a partir de los cuales establecer el paso de la alfabetización a la adquisición de cultura científica en un sentido amplio; por tanto, es necesario rescatar la concepción de que los sistemas educativos tienen como objetivo, no solo enseñar conceptos, sino formar ciudadanos.

\section{CONCLUSIONES}

Del análisis y discusión presentados en el trabajo se pueden extraer algunas conclusiones principales:

1.- Existe un desajuste significativo entre las expectativas creadas por las iniciativas políticas e institucionales adoptadas con la finalidad de mejorar la calidad y rendimiento de la educación científico-tecnológica en Iberoamérica; un desajuste que está estrechamente vinculado al modelo positivista que aún subyace a la enseñanza de las ciencias en los contextos educativos.

2.- Siguiendo a los autores más representativos que dentro de la tradición CTS han abordado el análisis de la ciencia y la tecnología contemporáneas, se ha constatado cómo, especialmente a partir de los años 80 del siglo XX, estas han sido objeto de un cambio significativo en su dinámica interna, que tiene consecuencias relevantes para la producción misma del conocimiento científicotecnológico.

3.- Las características más significativas que vienen a describir la dinámica científica y tecnológica a partir de la identificación de ese nuevo modo de producción de conocimiento, así como de la crítica a la concepción positivista de la ciencia y la tecnología iniciada ya dos décadas antes desde los estudios CTS, son aquellas relativas a su carácter social, la presencia de incertidumbre y riesgos, el disenso entre expertos, la inclusión de los aspectos éticos y valorativos y la vinculación con la política.

4.- Se ha mostrado que, como consecuencia de las aportaciones del enfoque CTS, el paradigma de cultura científica ha sido a su vez modificado, pasando desde su identificación con la alfabetización -ligada a la concepción positivista de la ciencia- a una noción más amplia que incluye actualmente los aspectos sociales, comportamentales y actitudinales.

5.- Se han analizado los hitos más significativos en los tres modelos fundamentales bajo los cuales se ha venido describiendo la cultura científica: alfabetización, public understanding of science, ciencia y sociedad.

6.- Se ha examinado la realidad del marco educativo iberoamericano relativo a la enseñanza de la ciencia y la tecnología, comprobando que no se ha producido en ellos una evolución similar a la que ha tenido lugar en los otros dos ámbitos tratados (a saber, la ciencia y la tecnología y la cultura científica). 
7.- La ciencia y la tecnología que se presentan en las aulas continúan ciñéndose al prisma positivista (conocimientos terminados, valorativamente neutrales, etc.) y con un objetivo meramente alfabetizador, atendiendo, fundamentalmente, a las teorías y conceptos.

8.- La ciencia y la tecnología que son habitualmente enseñadas no tienen como horizonte la generación de cultura científica, entendida en el sentido amplio que se ha presentado y que responde de manera más adecuada a la realidad y necesidades contemporáneas.

9.- Se propone sustituir el paradigma de la alfabetización científica, ya obsoleto, por el de una cultura científica que permita una aprehensión adecuada, crítica y eficaz de los conocimientos, convirtiéndolos, de este modo, en una herramienta operativa tanto dentro como fuera de los contextos educativos.

\section{AGRADECIMIENTOS}

Agradecemos el apoyo, para la realización de este trabajo, del proyecto Concepto y dimensiones de la cultura científica del Ministerio de Ciencia e Innovación del Gobierno de España y el Programa "Severo Ochoa" de becas predoctorales de la Fundación para el Fomento en Asturias de la Investigación Científica Aplicada y la Tecnología (FICYT) del Principado de Asturias.

\section{REFERENCIAS}

Abell, S.K. y N.G. Lederman, Handbook of Research in Science Education, Lawrence Erlbaum Associates, New Jersey, Estados Unidos (2007).

Bauer, M.W., N. Allum y S. Miller, What can we learn of 25 years of PUS survey research? Liberating and expanding the agenda, Public Understanding of Science, 16, 79-95 (2007).

Beck, U., Risikogesellschaft - Auf dem Weg in eine andere Moderne, Suhrkamp, Frankfurt, Alemania (1986).

Cámara, M. y J.A. López, Dimensiones políticas de la cultura científica, en Apropiación social de la ciencia, 63-89, Biblioteca Nueva, Madrid, España (2008).

De Arriba, D. Educar para participar en educación de personas adultas mediante una alfabetización científica con orientación CTS, Revista Iberoamericana de Educación, 44 (2), 1-7 (2007).

Duschl, R. Science Education in Three-Part Harmony: Balancing Conceptual, Epistemic, and Social Learning Goals, Review of Research in Education 32, 268-291, (2008).

Durant, J. A new agenda for the public understandig of science. Inaugural Lecture., Imperial College, Londres, Reino Unido (1995).

Echeverría, J. La revolución tecnocientífica. Fondo de Cultura Económica, Madrid, España (2003).

Edge, D. Reinventing the Wheel, en Handbook of Science and Technology Studies, 3-23, Sage Publications, California, Estados Unidos (1995).

FECYT, OEI, RICYT. Cultura científica en Iberoamérica. Encuesta en grandes núcleos urbanos. FECYT, OEI, RICYT, Madrid, España (2009).

Funtowicz, S.O. y J.R. Ravetz, Science for the post normal age, Futures, 25, 739-755 (1993).

Godin, B. y Y. Gingras. What is a scientific and technological cultura and how is it measured?, Public Understanding of Science, 9, 43-58 (2000).

Gibbons, M., C. Limoges, H. Nowotny, S. Schwartzman, P. Scott, M. Trow. The new production of knowledge: the dynamics of science and research in contemporary societies, SAGE Publications, Londres, Reino Unido (1994).

Holbrook, J. y Rannikmae, M., The meaning of scientific literacy, International Journal of Environmental and Science Education (IJESE), 4(3), 275-288 (2009) 
Howe, K.R., Positivist-Dogmas, Rhetoric, and the Education Science Question, Educational Researcher, 38, 428-440 (2009).

Jasanof, S. Procedural Choices in Regulatory Science, Technology in Science, 17 (3), 279-293 (1995).

Kuhn, T. The Structure of Scientific Revolutions, University of Chicago Press, Chicago, Estados Unidos (1962).

LOGSE, Ley Orgánica 1/1990, de 3 de octubre, de Ordenación General del Sistema Educativo, Madrid, España (1990), http://www.boe.es/boe/dias/1990/10/04/pdfs/A28927-28942.pdf. Acceso: 3 de febrero de 2011.

LOE, Ley Orgánica 2/2006, de 3 de mayo, de Educación, Madrid, España (2006), http://www.boe.es/boe/dias/2006/05/04/pdfs/A17158-17207.pdf. Acceso: 3 de febrero de 2011.

López, J.A. Prólogo, en Papeles Iberoamericanos: Educar para participar. Materiales para la educación CTS, OEI, Madrid, España (2005).

López, J.A. y M. Cámara, La cultura científica en España, en El español, lengua para la ciencia y la tecnología: presente y perspectivas de futuro, Inst. Cervantes, Madrid, España, 17-40 (2009).

Mansour, N. Science-Technology-Society (STS). A New Paradigm in Science Education, Bulletin of Science, Technology \& Society, 29 (4), 287-297 (2009).

Martín, M. (ed.). Educación, ciencia, tecnología y sociedad. Documentos de trabajo, nº 3, CAEUOEl, Madrid, España (2009).

Miller, J. D. The Development of Civic Scientific Literacy in the United States, en Kumar y Chubin (eds.). Science, Tecnology and Society, Kluwer Academic/Plenum Pub., New York, USA (2000)

OCDE. Informe PISA 2006. Competencias científicas para el mundo de mañana, Santillana, Madrid, España (2008).

OEI, Metas educativas 2021. La educación que queremos para la generación de los bicentenarios, OEl, Madrid, España (2010).

Putnam, H. What Theories are Not, en Logic, Methodology and Philosphy of Science, 240-252, Stanford University Press, Stanford, Estados Unidos (1962)

Royal Society. The public understanding of science, Royal Society, Londres, Reino Unido (1985).

Shen, B. Science Literacy, American Scientist, 63 (3), 265-268 (1975).

Snow, C.P. The two cultures and the scientific revolution, Cambridge University Press, Cambridge, Reino Unido (1959).

Sturgis, P.J. y N.C. Allum. Science in Society: Re-evaluating the Deficit Model of Public Attitudes, Public Understanding of Science, 13, 55-74 (2004).

UE. Biotechnology and Genetic Engineering, Comisión Europea, Inra, Francia (1993). http://ec.europa.eu/public_opinion/archives/ebs/ebs_080_en.pdf. Acceso 3 de febrero de 2011.

UE. Proyecto Europa 2030. Retos y oportunidades. Informe al Consejo Europeo del Grupo de Reflexión sobre el futuro de la UE en 2030, Unión Europea, Stuttgart, Alemania (2010), http://www.consilium.europa.eu/uedocs/cmsUpload/es_web.pdf. Acceso: 3 de febrero de 2011.

UNESCO. Declaración sobre la ciencia y el uso del saber científico. Adoptada por la Conferencia mundial sobre la ciencia el $1^{\circ}$ de julio de 1999, Budapest, Hungría (1999).

Weinberg, A.M. Science and Trans-Science, Minerva, 10, 209-222 (1972).

Winner, L. The whale and the reactor: a search for limits in an age of high technology, University of Chicago Press, Chicago, Estados Unidos (1986).

Wynne, B. Public Understanding of Science, en S. Jasanoff, G.E. Markel, J.C. Petersen and T. Pinch (eds.), Handbook of Science and Technology Studies, SAGE, Londres, UK, 361-88 (1995).

Ziman, J. ¿Qué es la ciencia? Cambridge Univeristy Press, Madrid, España (2003). 